\title{
Opinion
}

\section{The Phenotype Landscape of Cancer in the Genotype Era}

\author{
Daniel Gandia, MD* \\ Hematology and Oncology, Medical Strategy \& Science, Therapeutic Science \& Strategy Unit, Ing. Butty $2759^{\text {th }}, \mathrm{Cl} 00 \mathrm{I}$ CABA, Argentina \\ "Corresponding author \\ Daniel Gandia, MD \\ Clinical Oncologist, Hematology and Oncology, Medical Strategy \& Science, Therapeutic Science \& Strategy Unit, Ing. Butty $2759^{\text {th }}$, CI00I CABA,Argentina; \\ Office.54II 4I32 65 I6; Mobile.549II 6496 5444; E-mail: Daniel.Gandia@iqvia.com
}

\section{Article information}

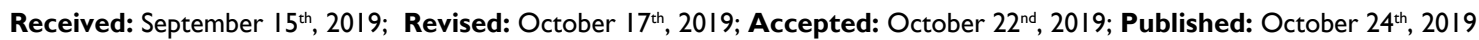

Cite this article

Gandia D. The phenotype landscape of cancer in the genotype era. Cancer Stud Mol Med Open J. 2019; 5(I): 26-27. doi: 10.17।40/CSMMOJ-5-I29

ome tumor types such as testicular cancer, leukemia and lym$\checkmark$ phomas, have their origin in cells that comport a common embryonic origin. This phenomenon was "unconsciously" the roadmap to treat and cure them by classic chemotherapy. The cells are very chemosensitive indeed as they are "immature" in origin.

Chemotherapy has also positively impacted the overall survival of some pediatric and adolescent cancers (leukemias, lymphomas, bone tumors) and in many other adult ones such as breast, ovarian, head and neck and colorectal cancers. Despite everything, we also face other difficult to treat cancers which remain chemo resistant (lung, melanomas, pancreatic, biliary tract cancers as the most representative ones).

As basic and translational cancer researches evolve, these tumor-types have turned into a significant focal point of intrigue. So, what to do in order to work-out this cancer resistance dilemma? Targeting some tumor genetic mutations such as those of the tyrosine kinase types, oncogene- driven growth factor receptors and some immune molecules such as those of the programmed-death pathway (PD-1 and PD-L1) and the cytotoxic T-lymphocyte-associated protein 4 (CTLA4) axis, enable us to battle the malignant growth.

Regarding these aforementioned cellular pathways, drugs which are of small molecules, target the first two druggable ones and treat lung, renal and malignant melanoma. The third one, "the mammoths": monoclonal antibodies (MABs), can disrupt angiogenic pathways, block some oncogenic drivers (such as RAS) and mainly deblock the lazy T-lymphocytes by allowing them to "eat tumors" (checkpoint inhibitors to treat melanoma, lung, squamous head and neck tumors). This last one, became the treatment of cancer by the inhibition of negative immune regulation for which the researchers have been granted Nobel prize. ${ }^{1}$
But, all the before-mentioned is a "partial win" in the treatment of cancers. There will be still continued exploring "for more and better" in the above-targeted therapy.

The answer for therapy failures is related to the old concept of tumor heterogeneity. Imagine a view with the light microscope, where the tumor sample even seeming homogeneous, is composed of "cells of different imaginable colors". Tumor cell phenotype heterogeneity is the main cause of secondary resistance and also of progression in the already mentioned chemosensitive tumors, and one of the main causes of the non-tumor responses in the less de novo chemosensitive ones such as lung cancer.

Nowadays, tumor heterogeneity explains why therapy targeted to a driver gene or protein in a tumor is met with disease progression, the disease at some point now it has a new driver gene in some cells, or more easily the emerging presence of a new clonal cell population. So, is the answer we need to explore for new biomarkers? Yes or no.

Tumor heterogeneity is something more than this, it's mainly the cellular dynamics between the different tumor clones that lead to resistance. These dynamics are essential to the phenotypic landscape of the cancer cell populations. Tumor cells have a "great talk" between them (growth factors signals). They have cooperation within the different clones: two clones evolve complementary traits. Even though each clone is not self-sufficient, their cooperation results in malignancy.

They have competition for oxygen and substrates, and also interactions with the mesenchyme in the case of epithelial tumors. In this sense, clonal lineages with similar fitness may coexist and compete within a tumor, a phenomenon known as clonal interference. This adds a higher complexity to the cancer-disease 
process. In origin, cancer is a one-cell-disease but, it is a tissue disrupted-related disease during the rest of its whole lifecycle. With the old, current and novel drug-tools, we must learn "how to treat the cancer phenotype in a better way." In the early days of chemo, in some way all the aforementioned have been taken into account: tumor cellular kinetics explained by the Goldie-Coldman model measures genetic instability to the probability of a tumor containing drug-resistant clones. This works as a framework for understanding some types of tumor cell kinetics and posterior chemo responses and resistance in them.

My opinion here is to highlight the tumor heterogeneity with its clonal dynamics as a whole: the Cancer Phenotype.

Cancer can also be considered as an evolutive Darwinian process, where the most adaptable cancer cells within a heterogeneous population survives, proliferate and invade other organsites. Phenotype plasticity is an enabler of tumor progression and therapy resistance. Within it, is tumor heterogeneity what makes malignancies as the "masters of evasion".

On the other hand the tumor immunophysiology, the scenario is even more complex in its phenotypic assessment, with different cancer-immune phenotypes, for e.g., inflamed-tumor, immune-excluded tumor and immune-desert tumor.

Each is associated with specific underlying biological mechanisms that may prevent the host's immune response from eradicating the tumor. Similarly, an immune-desert phenotype can be the result of immunological ignorance, the induction of tolerance or a lack of appropriate T-cell priming or activation. Immune-excluded tumors may reflect a specific chemokine state, the presence of particular vascular factors or barriers, or specific stromal-based inhibition. Finally, inflamed tumors can demonstrate infiltration by a number of subtypes of immune cells (T-cells, myeloid-derived suppressor cells, suppressor B-cells and cancer-associated fibroblasts such as in Melanoma).

Finally, for some difficult to treat cancer scenarios, where the disease has a progression over progressions or are directly refractories ones, the medical therapy to be utilized are in an adaptive or metronomic manner, directed to some tumor cell populations while keeping up calm on others with the lesser growth (e.g., mesothelioma, pancreatic carcinomas, biliary tract cancers mainly).

Some cancers can be considered as chronic diseases. As a physician, the "primum non-nocere" concept is essential: "to treat the patient, not just the cancer".

\section{REFERENCES}

1. Noberforsamlingen: The Nobel Assembly at Karolinska. Web site. https://www.nobelprize.org/uploads/2018/10/press-medicine2018.pdf. Accesssed September 14, 2019. 